\title{
UPGRADE PLANS OF THE VACUUM SYSTEM OF THE ESRF
}

\author{
R. Kersevan ${ }^{\dagger}$, L. Goirand, ESRF, Grenoble, France.
}

\begin{abstract}
The ESRF has been delivering beams to users for over 12 years. The performance of the storage ring has surpassed the original specifications with respect to many accelerator parameters, such as stored current, emittance, beam stability, beam availability and so on. Along the years, many of its sub-systems have been improved in order to cope with these more demanding conditions. New experimental techniques and arrangements, such as nanofocusing on the samples, call for an important upgrade of the machine and the beamlines. Such an upgrade will also allow the ESRF to stay at the forefront of synchrotron radiation research. A conceptual design report for such ESRF upgrade is under preparation. This paper details the current plans for the upgrade of the storage ring vacuum system.
\end{abstract}

\section{THE UPGRADE STRATEGY}

Very recently, the ESRF Council has been presented with a preliminary version of the so called Purple Book (PB) [1], to be finalized and discussed again at future Council meetings. The PB details the current state of the proposals for upgrading the accelerator and sources, the beamlines (BLs) and all infrastructures on site which are necessary for improving the scientific output of the laboratory. Accelerator and source developments will allow an increase in brilliance, higher X-ray fluxes, a larger number of BLs, improved stability of the photon beams, increased flexibility of the insertion devices (IDs). All these improvements will require a modification of the vacuum system, in particular in the areas of the ID straight sections and the dipole and crotch absorbers. Some preliminary tests implementing different lattices have been already reported [1,2]. These and more recent tests have confirmed that longer ID straight sections could be implemented in a rather straightforward way, by modifying 2 girders, removing 4 quadrupoles and installing 2 new quadrupoles in each cell. The effective length available for the installation of ID segments would go from the present day 5 meters up to 7 . These longer sections could be used either to implement a $7 \mathrm{~m}$ long ID split with 4 segments, or to implement a canted-ID scheme whereby a beam trajectory chicane takes place by using 3 steerers. The two sections of the canted-ID straight, would be characterized by a $2 \times 2.7 \mathrm{mrad}$ horizontal separation, allowing for independent front-ends (FEs) and BL hutches. This horizontal separation would also entail a re-design of the dipole and crotch absorber areas. The latter need also to be modified in order to cope with the increased power densities generated by the lower emittance beams, and possible current upgrades.

\footnotetext{
${ }^{\dagger}$ Corresponding author
}

\section{VACUUM SYSTEM}

A standard cell at the ESRF has two dipoles, which for cost reasons would be reused, as would most of the sextupoles and quadrupoles. Considering that the tunability of the synchrotron radiation (SR) comes exclusively from the use of IDs, while the bending magnet (BM) spectrum is fixed for a given energy, it is the straight sections where the IDs are installed that need to be modified. This concerns almost $21 \mathrm{~m}$ out of 26.4 of each standard cell. The injection straight and the $3 \mathrm{RF}$ cavity straights are dealt with differently.

\section{Narrow-gap Chambers}

Nowadays, the ESRF is equipped with 9 in-vacuum and 26 in-air narrow gap chambers. The large majority of the narrow gap chambers are $5 \mathrm{~m}$ long with external vertical apertures of $10 \mathrm{~mm}$ [3]. A $5 \mathrm{~m}$ long ID chamber would be increased to a $7 \mathrm{~m}$ length. The present design employs extruded-aluminum chambers which are coated internally on ESRF premises with non-evaporable getter (NEG) materials [4]. Their beam chamber cross-section is elliptical, 57x8 $\mathrm{mm}^{2}(\mathrm{HxV})$. Ray-tracing analysis has shown that this size would be too small in case of a canted-ID set-up, and therefore an increased horizontal size of $67 \mathrm{~mm}$ is under consideration. Although the NEG coating has proved to be very effective at reducing the pressure bump along the $5 \mathrm{~m}$ long ID chambers, it is envisaged to improve the pumping along the $7 \mathrm{~m}$ long version by adding a small ion-pump in the middle of the chamber. The pump would be connected to the extrusion by means of explosion-bonding Al-stainless steel (SS) flange, and 4 small $6 \mathrm{~mm}$ internal diameter holes placed on the side of it. Although the conductance of such holes has been calculated to be only $\sim 3.4 \mathrm{l} / \mathrm{s}$, the effect on the pressure profile along the chamber seems to be quite large. Figure 1 shows the comparison of a $7 \mathrm{~m}$ long ID chamber vs a $5 \mathrm{~m}$ long one with and without such pumping in the middle. The different outgassing yield shapes are due to the fact that the $7 \mathrm{~m}$ long chamber considered here would be the one used for a canted-ID, for which the angle of incidence changes in the middle due to the chicane [1, 2]. Figure 2 shows some details of an early design of the mid-ID pumping geometry, which has recently been optimized. The exact size and shape chosen for the mid-ID chicane magnet, under final design by the ESRF ID group, will determine the connection and support for the small ion-pump placed in the middle position. Coating of the $7 \mathrm{~m}$ long ID chamber will need a refurbishment of our NEG-coating facility, as $\sim 6 \mathrm{~m}$ is the maximum length of the chambers we can coat while in a vertical position [4]. In case a canted-ID solution is implemented, then the $7 \mathrm{~m}$ section could be split into 3 parts, upstream ID, mid-pumping and chicane section, 
and downstream ID, with maximum lengths of less than $3.5 \mathrm{~m}$, a length which can be handled easily by our two existing coating towers.

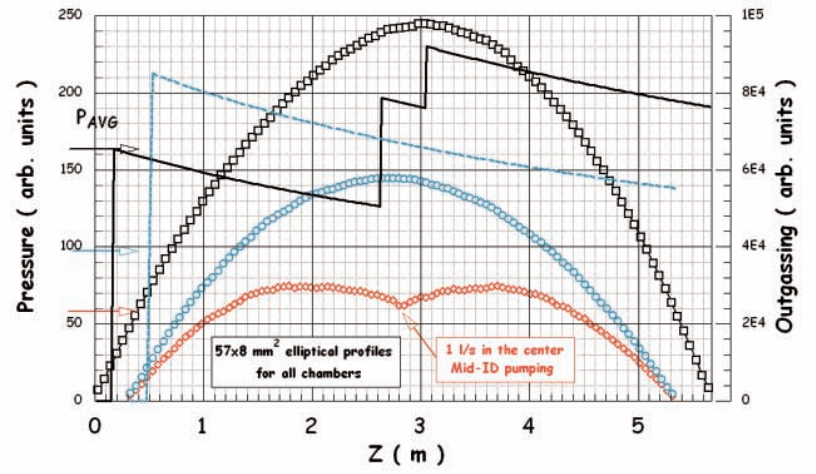

Figure 1: Comparison of the pressure profiles with and without mid-ID pumping for a $5 \mathrm{~m}$ long " $10 \mathrm{~mm}$ " chamber compared with a $7 \mathrm{~m}$ long one. Solid and dotted lines show the outgassing profiles generated by the BM placed upstream.

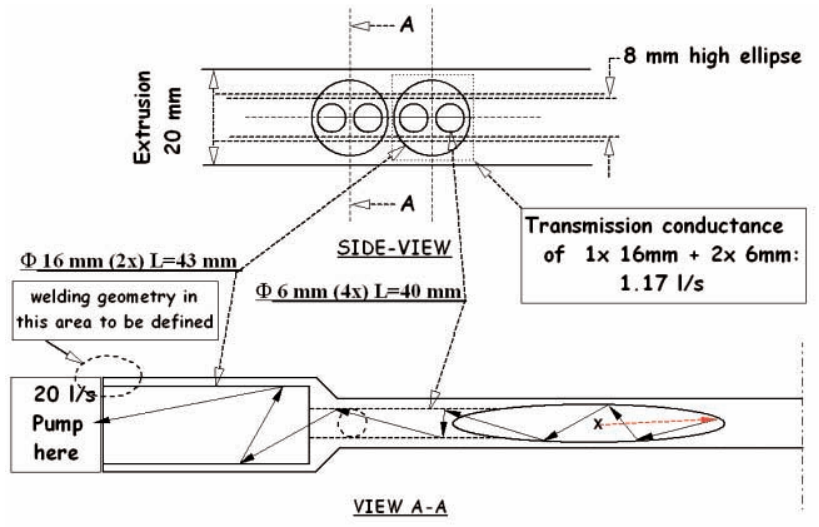

Figure 2: Mid-ID pumping concept.

\section{Quadrupole Chambers}

The fact that the ID sections become longer by $\sim 2 \mathrm{~m}$, means that the two quadrupole chambers placed upstream and downstream of the IDs will have to become correspondingly shorter by approximately $1 \mathrm{~m}$ each, going from a length of $3.5 \mathrm{~m}$ down to $2.5 \mathrm{~m}$. The latest version of the upgrade lattice would also call for new quadrupoles to be placed immediately before and after the ID section. These quadrupoles would have a $35 \mathrm{~T} / \mathrm{m}$ field gradient (compared to the present $19 \mathrm{~T} / \mathrm{m}$ ), and would not allow side-ports for pumps to be placed inside their yoke [1]. We have therefore proposed to use in these sections new extruded-aluminum chambers with NEG-coating. The inscribed diameter of the new quadrupoles being 56 $\mathrm{mm}$, a $68 \times 20 \mathrm{~mm}^{2}$ pseudo-ellipse (internal dimensions) has been envisaged, leaving space for the thickness of the chamber's wall and the bake-out heaters for NEG activation. The specific conductance of the $68 \times 20 \mathrm{~mm}^{2}$ pseudo-ellipse has been calculated to be $6.0 \mathrm{l} \cdot \mathrm{m} / \mathrm{s}$, much bigger than that of a $3.5 \mathrm{~m}$ long, 30x20 mm prototype which had been successfully tested on the storage ring in 2005. Its specific conductance was only $1.72 \mathrm{l} \cdot \mathrm{m} / \mathrm{s}$ [3].

\section{Dipole and Crotch Absorbers}

The envisaged improvement in emittance and the possibility of increasing the stored currents has lead us to review the design of the crotch absorbers. The present crotch absorbers are inserted vertically, and the surface hit by the X-rays is flat, vertical and inclined at an angle of the order of 20 degrees with respect to the incident X-ray beam. A new design of absorber is being prototyped that will sustain a higher heatload and will be simpler to manufacture. The water cooled surface is composed of teeth inclined in both the vertical and horizontal directions in order to spread the heat load. Such design is becoming standard in the recently built synchrotron sources. A view of the "V"-shaped teeth of the upper and lower jaws of the absorber is shown in Fig.3, together with the beam chamber and its pumping slots.

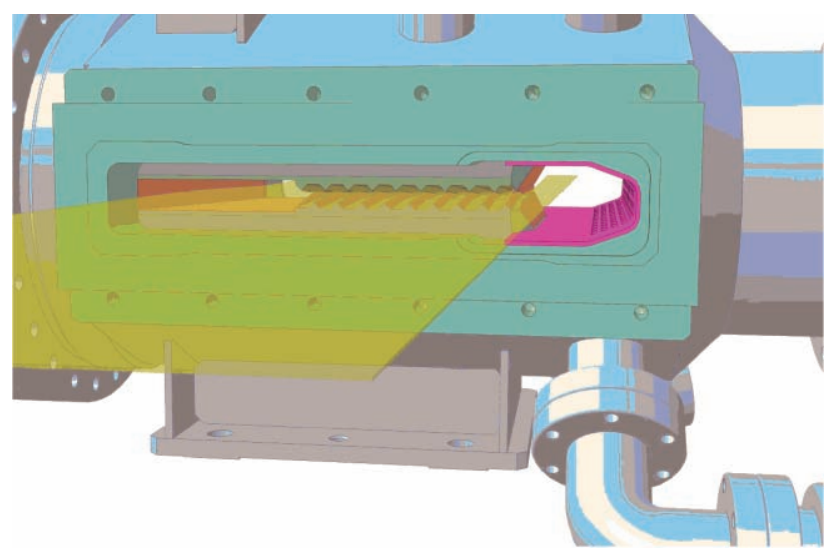

Figure 3: View of the new crotch absorber geometry, with interleaved "V"-shaped teeth. The SR fan is indicated in yellow. The beam chamber with its pumping slots is shown in pink. The main pumping system, a $500 \mathrm{l} / \mathrm{s}$ ionpump coupled with a NEG pump is attached on the right side. The rectangular flange on the front is clamped and welded to the dipole chamber, not shown here.

A detailed analysis of the expected pressure profile for this new configuration has been carried out, and compared to the existing one. The orientation of the new crotch absorber is such that the trapping efficiency of the ion/NEG pumps is lower than that of the present case, as shown in Fig.4. Particular attention has been paid to minimizing the pressure bump along the straight line of sight of the related BL, as this would potentially increase the amount of bremsstrahlung (BS) radiation (on this and related issues see for instance [6]). Fig. 4 shows that for the same $200 \mathrm{~mA}$ current the upgraded crotch would produce an average pressure along the dipole chamber $50 \%$ higher than for the present design. The fact that the new crotches are designed to withstand $300 \mathrm{~mA}$ beams, and that BS scales as the square of the current, leads us to the conclusion that we need to implement some sort of distributed pumping along the dipole chamber. The details of this have not been clarified yet, but they should not be too difficult to implement, as there is enough space to add a long pumping slot on the inside wall of the beam 
chamber, with some sort of NEG or distributed ionpumping along it.

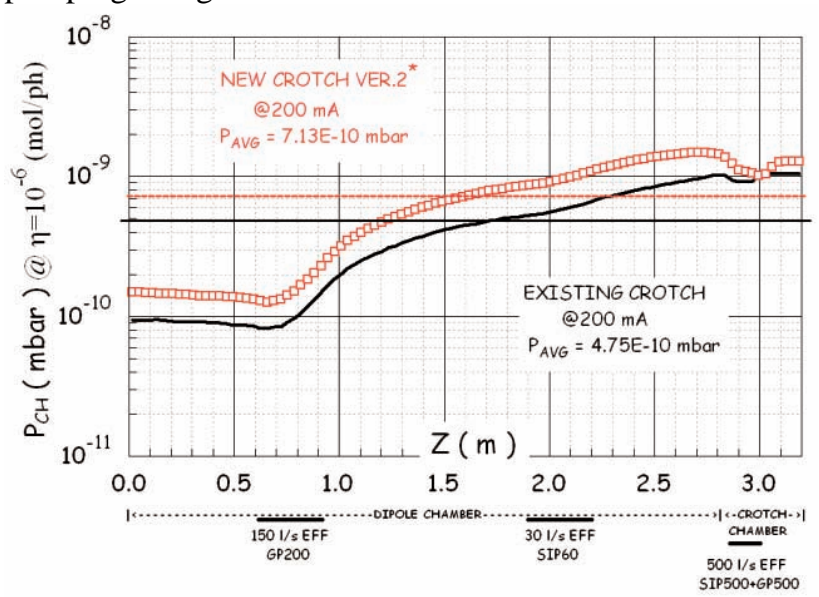

Figure 4: Pressure profiles along proposed dipole/crotch chambers, and comparison to existing case.

Preliminary calculations show that a modest linear pumping speed over the length of the dipole would be sufficient to bring the average pressure well below the value shown in Fig.4.

\section{RF Cavities}

As part of the ESRF upgrade, the ring current will be rised to $300 \mathrm{~mA}$. Such current can be achieved either using the existing 5-cell "LEP-type" RF cavities with the help of a bunch by bunch feedback presently under development or by using specifically designed RF cavities with strong Higher Order Mode damping (HOM).

The ESRF RF Group is actively collaborating with BESSY and ALBA, on the development of HOM-damped RF cavities. A $500 \mathrm{MHz}$ version exists already at the Willy Wien Metrology Light Source in Berlin, a similar one is under design at the Spanish light source ALBA, and a $352 \mathrm{MHz}$ one is being studied at the $\operatorname{ESRF}[1,5,8]$.

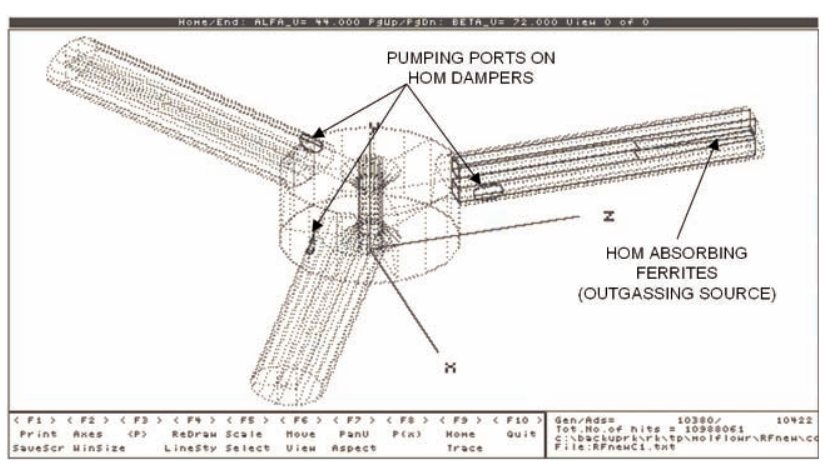

Figure 5: Re-touched screen shot of the 3D Molflow model used for the analysis of the pumping efficiency [7].

The chosen design is characterized by 3 long protruding arms, symmetrically placed around the single-cell cavity axis, used for feeding the RF power and for damping the unwanted HOMs. The ridged waveguides are terminated with water-cooled ferrite tiles, which are a source of gas desorption. A preliminary analysis has been carried out on the pumping efficiency of this design, which shows that installing the pumping along the 3 arms would help keep the pressure low inside the cavity, lower than by pumping along the beam chamber on either side of each cavity. Fig. 5 shows a view of the 3D Montecarlo model made to simulate the pumping efficiency (cavity/beam $\mathrm{Y}$ axis pointing upward).

\section{CONCLUSIONS}

Based on the extensive experience gained since more than 7 years on the use of NEG-coated chambers, the ESRF plans to develop further this technology and implement it for making $7 \mathrm{~m}$ long ID chambers and quadrupole chambers, minimizing the number of ion pumps and NEG pumps, their related probability of failure, and the well documented capital and operating costs due to the need of cabling and controlling them. New crotch absorbers capable of withstanding the power densities foreseen for the $300 \mathrm{~mA}$ beams and higher have been designed, and are under prototyping. A new design for the RF cavities is also under study, together with an optimization of its pumping system.

\section{ACKNOWLEDGMENTS}

The authors are indebted with the Vacuum and Mechanical Engineering Groups. Many thanks to our machine director Pascal Ellaume for reviewing the manuscript.

\section{REFERENCES}

[1] “Science Programme 2008-2017”, Vol.1 and 2, May 2007, unpublished, to be updated and published in late 2007.

[2] A. Ropert, L. Farvaque, "Optimisation of a New Lattice for the ESRF Storage Ring”, EPAC'06, Edinburgh, June 2006, p. 1942.

[3] M. Hahn, R. Kersevan, I. Parat, "Status report on the performance of NEG-Coated Chambers at the ESRF”, EPAC-06, Edinburgh, June 2006, p. 1420.

[4] R. Kersevan, "NEG-Coated Vacuum Chambers at the ESRF: Present Status and Future Plans", EPAC-02, Paris, p. 2565.

[5] J-L. Revol et al., "Operation and Recent Development at the ESRF”, EPAC'06, Edinburgh, June 2006, p. 3290.

[6] P. Berkvens, P. Colomp, R. Kersevan., Proceedings of RadSynch '07 conference, CLS, Saskatoon, 6-8 June 2007, to be published in Radiation Measurements.

[7] R. Kersevan, "Molflow User's Guide”, available from one of the authors (RK).

[8] N. Guillotin, J. Jacob, V. Serrieres, "Development of a HOM Damped Copper Cavity for the ESRF", EPAC’06, Edinburgh, June 2006, p. 1244. 